\title{
Effects of water droplet breakup on hydrogen/air detonations
}

\author{
Yong Xu, Majie Zhao, Huangwei Zhang* \\ Department of Mechanical engineering, National University of Singapore, Singapore \\ *Corresponding author email: huangwei.zhang@nus.edu.sg
}

\begin{abstract}
Two-dimensional numerical simulations with Eulerian-Lagrangian method are conducted to study the influence of fine water droplet breakup on detonation propagation in stoichiometric hydrogen/air mixture. General features of gas phase and water droplets are well captured. Numerical soot foils are used to characterize the influence of droplet breakup on the detonation wave, and the results confirm that droplet disintegration make propagation detonation wave more stable under the studied conditions. Analysis on unsteady detonation propagation process is performed with the evolutions of spatial distribution of interphase transfer rates (mass, energy, and momentum). The interactions between detonation wave and water droplets demonstrate that the breakup model have more pronounced influence on mass and energy transfer than the momentum transfer. Moreover, high interphase transfer rates of disintegration case are observed at the onset of detonation propagation, and they gradually decrease. It is concluded that inclusion of droplet breakup significantly alters propagation and two-phase coupling of the gaseous detonation in fine water mists.
\end{abstract}

\section{Keywords}

Hydrogen detonation; droplet breakup; water droplets; Eulerian-Lagrangian method

\section{Introduction}

There are increased interests in exploring effective approaches to mitigate detonation of flammable gas, related to prevention from explosion hazards and industrial safety assessment $[1,2]$. Water is an ideal detonation mitigant due to numerous advantages [3,4]. Specifically, it can absorb considerable heat from gas phase due to large heat capacity and latent heat of evaporation [5]. Also, it is readily available with low cost and lots of flexibilities. Meanwhile, use of water would not bring environmental pollution. Moreover, as a solution, it is possible to include proper additives, e.g., alkali salts $(\mathrm{KCl}$ and $\mathrm{NaCl})$ [6], for better explosion inhibition. There are various forms of water utilized for detonation or explosion mitigation $[5,7,8]$, e.g., solid jet, diffuse jet and water mists. The last one is most promising since sprayed water droplets have large specific surface area and low terminal velocity, which allow them to continuously circulate in the explosion area in a manner of a total flooding gas. It therefore can effectively weaken the blast, inhibit chemical reaction, and reduce gas temperature. Although it has been widely used in various areas, e.g., process and energy industries, nuclear power plants, and even space applications, however, the mechanisms behind detonation / explosion inhibition with water mists are still not well understood.

There have been a series of studies about propagation of shock / blast waves in water sprays. For instance, Jourdan et al. [9] use water aerosol shock tube experiments to study shock attenuation in a cloud of water droplets. They characterize shock attenuation with shock tube (i.e., cross-sectional area) and droplet properties (e.g., total water volume, droplet size, loading rate and droplet specific surface area). With the similar experimental conditions, Chauvin et al. [10] find the peculiar pressure evolution after the transmitted shock wave in twophase mixture and they also measure the overpressures under different water spray conditions. Moreover, Adiga et al. [11] unveil the physical picture of fine water droplet breakup 
in detonation process and quantify the droplet fragmentation with breakup energy. Eulerian-Lagrangian simulations by Ananth et al. [12] are performed to examine the effects of mono-dispersed fine water mist on a confined blast. It is found that the latent heat absorption is dominant for blast mitigation, followed by convective heat transfer and momentum exchange. Furthermore, Schwer and Kailasanath [13] simulate unconfined explosions in water sprays, and find that the water mists can dampen the shock through vaporization and momentum extraction. Different from the observations by Ananth et al. [12], they claim that the momentum extraction plays a more important role in weakening the blast.

In the abovementioned studies, the effects of water mists on chemical reactions are not discussed, since they use air as the carrier gas (e.g., $[9,10])$, specify the chemically equilibrium gas from an explosion (e.g., [12]), or there is no direct interaction between water droplets and post-shock reaction zone (e.g. [13]). It is well known that detonation is a complex of coupled shock and reaction fronts, and therefore additional complexities may arise in droplet-detonation interactions. Thomas et al. [14] experimentally study detonations of hydrogen, ethane and acetylene with water sprays in a vertical tube. They attribute detonation failure to high heat loss due to water droplets compared to the combustion heat release. They also find that the water droplet size and loading densities are crucial to quenching a detonation. It is observed by Niedzielska et al. [15] that small (215 $\mu \mathrm{m}$ in their experiments) droplets with fast evaporation rate has strong influence on detonation quenching. Moreover, from detonation tube experiments, Jarsalé et al. [16] observe that presence of water spray drastically alters the detonation cell size, but the ratio of the hydrodynamic thickness to the cell size remain constant, regardless of water droplet addition. The effects of water mists on Deflagration-to-Detonation Transition (DDT) are also demonstrated with reduced overpressure and delayed timing of detonation development [8].

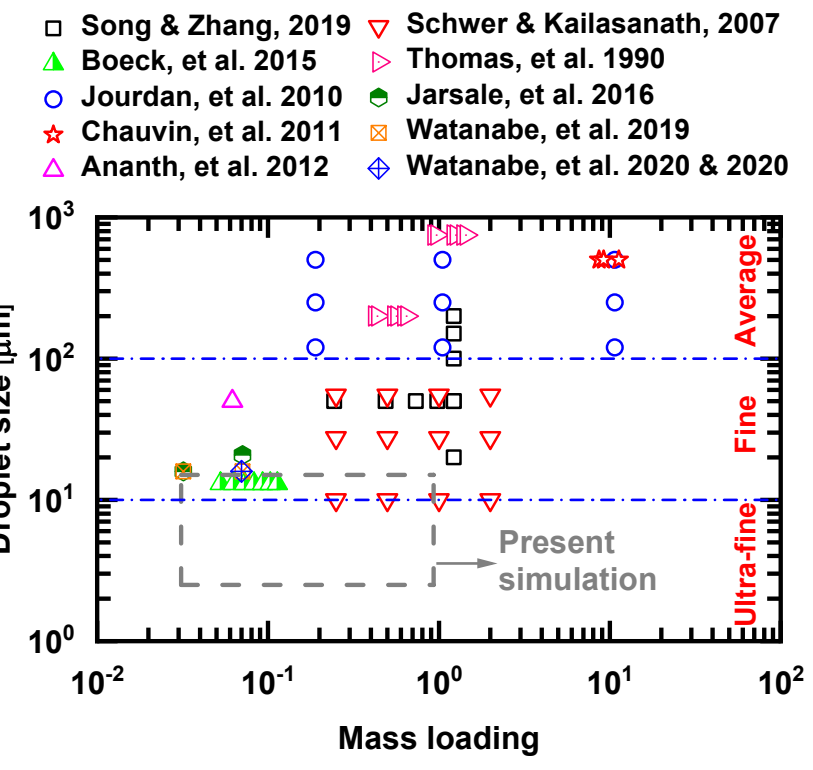

Figure 1 Studies of detonation and shock in water sprays. Droplet diameter spectrum (dashdotted lines) follows Grant et al. [5]. Average: droplet diameter range most relevant for firefighting. 
can see that most of the droplet diameters are above $20 \mu \mathrm{m}$, corresponding to the mass loading of 0.03-13.3. It is well known that fine or ultra-fine water droplets with diameter less than $20 \mu \mathrm{m}$ have outstanding performance in fire suppression, due to fast evaporation rate and high specific surface area [3,4]. Nevertheless, their effectiveness and the critical spray properties for detonation extinction and how the sprayed droplets interact with the detonation have not been reported yet. Four different droplet sizes: $2.5,5,10$, and $15 \mu \mathrm{m}$ are used in simulation. Only the results of droplet size $10 \mu \mathrm{m}$ are presented in this manuscript. Also, the polydispersed droplets are not considered.

Besides the foregoing experimental work [8,14-16], recent computational efforts provide us more insights on detonation in water sprays. For example, Song and Zhang simulate the methane detonation and find that the inhibition effects of water sprays are mainly reflected in reduction of flame temperature [7]. Watanabe et al. observe that the dispersed water droplets significantly alter the detonation flow field, and water droplet evaporation mainly occurs around $10 \mathrm{~mm}$ behind the leading shock [17]. Furthermore, the cellular patterns of dilute water spray detonation are more regular than those of the droplet-free detonations [18]. The interactions between detonation wave and water droplets change the hydrodynamic thickness. Exchange of mass, momentum and energy between two phases occurs within the hydrodynamic thickness, which lowers the detonation velocity and fluctuations downstream of the leading shock front. Their results also show that droplet breakup mainly occurs near the shock front [19], and the average diameter of the disintegrated water droplets is independent on the initial propagation velocity of the shock front. However, in these numerical studies, the influence of water droplet breakup on detonation propagation are not well understood.

\section{Governing equation and computational method}

The governing equations of mass, momentum, energy, and species mass fraction are solved with the ideal gas equation of state. The Lagrangian method is used to model the dispersed liquid phase, which is composed of a large number of spherical droplets [20]. The interactions between the droplets are neglected because we only study the dilute water sprays with the initial droplet volume fraction being generally less than $1 \%$ [21]. The vapor mass flux from the droplet into the gas phase is estimated following Refs. [22,23]. Only the Stokes drag is considered [24], and the convective heat transfer is modelled using the Ranz-Marshall correlation [25]. The droplet breakup model used in our simulation can be found in Ref. [26], and the maximum stable diameter determined by stability criterion and the total breakup time follows Pilch and Erdman [26]. Computational parcel is used in our simulations to represent a series of water droplets with similar properties (e.g., temperature, diameter, and movement). The gas and liquid phase governing equations are solved by a compressible two-phase reacting flow solver, $R Y$ rhoCentralFoam [27], which has been successfully applied for various detonation problems [28-32]. The hydrogen mechanism with 9 species and 19 reactions [33] is used, which is validated against the measured ignition delay and detonation cell size [34]. For the liquid phase, the droplets are tracked based on their barycentric coordinates. More detailed information about the numerical methods for gas and liquid phases can be found in Ref. [27]. 


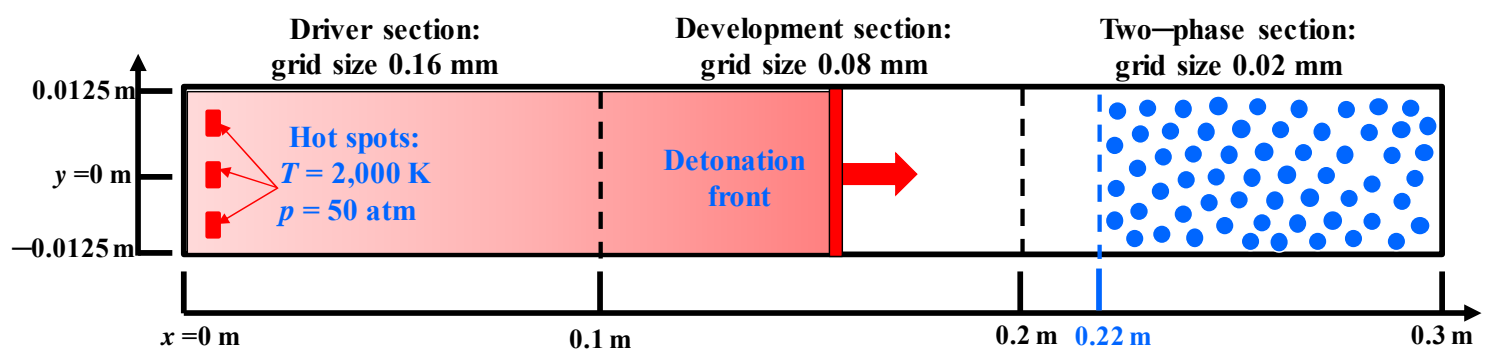

Figure 2. Schematic of the computational domain. Blue dots: water droplets.

\section{Physical model}

The computational domain is shown in Figure 2. The length ( $x$-direction) and width $(y-$ direction) are $0.3 \mathrm{~m}$ and $0.025 \mathrm{~m}$, respectively. It includes driver, development, and two-phase sections, as marked in Figure 2. They are initially filled with stoichiometric $\mathrm{H}_{2}$ /air premixture, with temperature and pressure being $T_{0}=300 \mathrm{~K}$ and $p_{0}=50 \mathrm{kPa}$, respectively. The HalfReaction Length (HRL) estimated from the purely gaseous ZND structure of $\mathrm{H}_{2}$ /air detonation is about $309 \mu \mathrm{m}$. Therefore, the resolution in the two-phase section is approximately 15 cells per HRL. The total length of the driver and development sections is about $647 \mathrm{HRL}$, and hence sufficient to minimize the detonation initiation effects before the detonation wave is transmitted into the two-phase section [35]. The upper and lower boundaries of the domain in Figure 2 are assumed to be periodic. For the left boundary $(x=0)$, the non-reflective condition is enforced for the pressure, while the zero gradient condition for other quantities [36]. Since the gas before the detonation wave is static, the boundary condition at $x=0.3 \mathrm{~m}$ is not relevant and in our simulations zero gradient conditions are assumed [37].

\section{Results and Discussion}

Available experiment results related to our simulation study are scarce. But the EulerLagrangian solver mentioned in the section: Governing equation and computational method have been validated. In this section, the case: initial droplet diameter $d_{d}^{0}=10 \mu \mathrm{m}$ and mass loading $z=0.31$ are analysed. The mass loading $z$ is estimated as the ratio of the total water mass to the mass (or volume) of the gaseous mixture within the droplet-containing region [21]. The density-weighted average interphase transfer rates are used to clarify the effects of water droplet breakup on hydrogen detonation. 

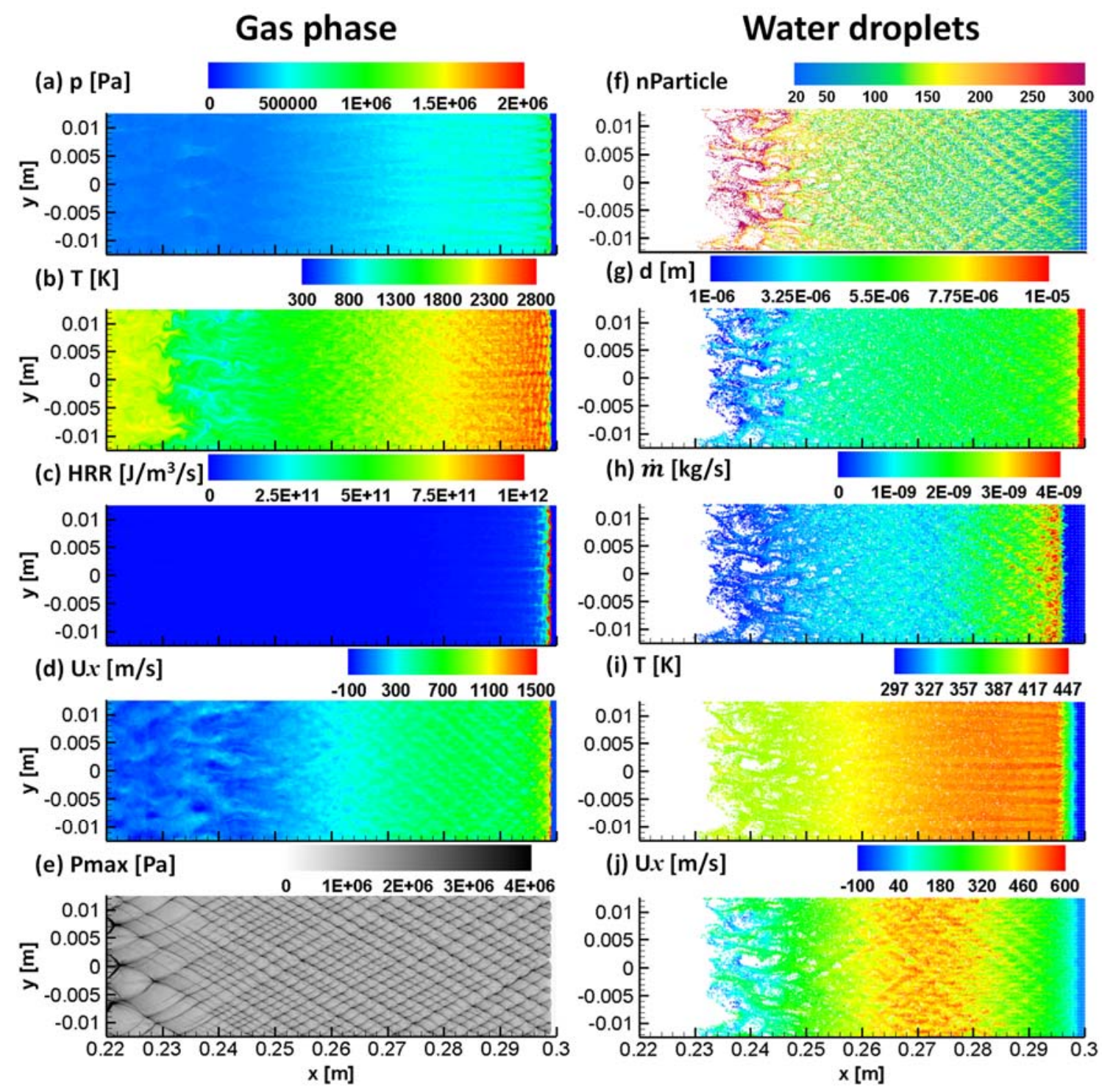

(h) $\dot{m}[\mathrm{~kg} / \mathrm{s}]$

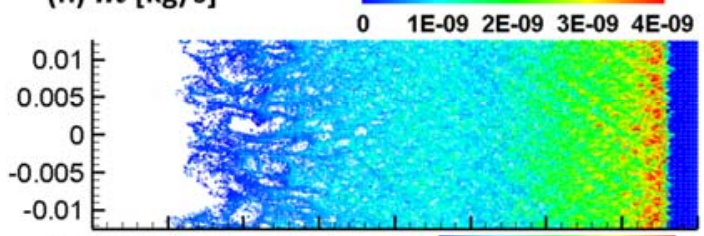

(i) $\mathrm{T}[\mathrm{K}]$

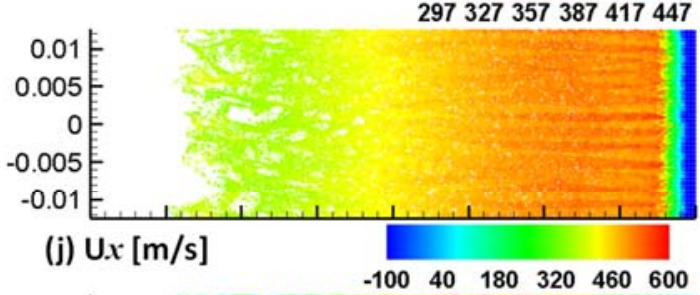

Figure 3. Flow fields of gas phase: (a) pressure, (b) temperature, (c) heat release rate, (d) velocity and (e) maximum pressure history; water droplets: (f) particle per parcel, (g) diameter, (h) evaporation rate, (i) temperature and (j) velocity. $d_{d}^{0}=10 \mu \mathrm{m}$ and $\mathrm{z}=0.31$.

The instantaneous flow fields of gas phase and water droplets are depicted in Figure 3. Multiple heads structure followed by transverse waves are found in Figure 3(a), and this indicates that the detonation waves are able to stable propagation in the two-phase section. The gas phase is heated to $2800 \mathrm{~K}$ by the detonative combustion heat release behind the shock front (Figure 3(c)), and then is gradually reduced due to the droplet cooling effect. The negative value of velocity in the downstream in Figure 3(d) implies that the momentum is transferred from gas phase to the water droplets. Trajectory of peak pressure in the detonation propagation under breakup process is recorded in Figure 3(e). Smaller and more regular cell size is formed in Figure 3(e), which means that the water droplet disintegration make propagation of detonation wave more stable compared with the initial period. For the water droplets, the number of particles per parcel in Figure 3(f) increases with the distance from the detonation wave as droplet breakup, and forward jet flows produced by the triple point collision, symbolled by the red strips, appear behind the shock front. The spatial distribution 
of particles per parcel is also evidenced by the droplet size reduction due to droplet breakup and evaporation. The water droplets are completely evaporated between $x=0.22-0.23 \mathrm{~m}$. Figure 3(h) reveals that larger evaporation rate occurs a short distance behind the shock front, which is corresponding to the droplet temperature field in Figure 3(i). The droplet temperature is increased to $447 \mathrm{~K}$ and strong droplet evaporation then occurs. The water droplets continue to evaporate in the post-detonation area until they are completely evaporated. However, the droplet velocity along the $x$-direction presents different distributions: it peaks between $x=0.26$ $-0.28 \mathrm{~m}$. This is caused by the delay of droplet acceleration, and after peak the droplet velocity is reduced as the gas velocity decrease in Figure $\mathbf{3}(\mathrm{d})$.
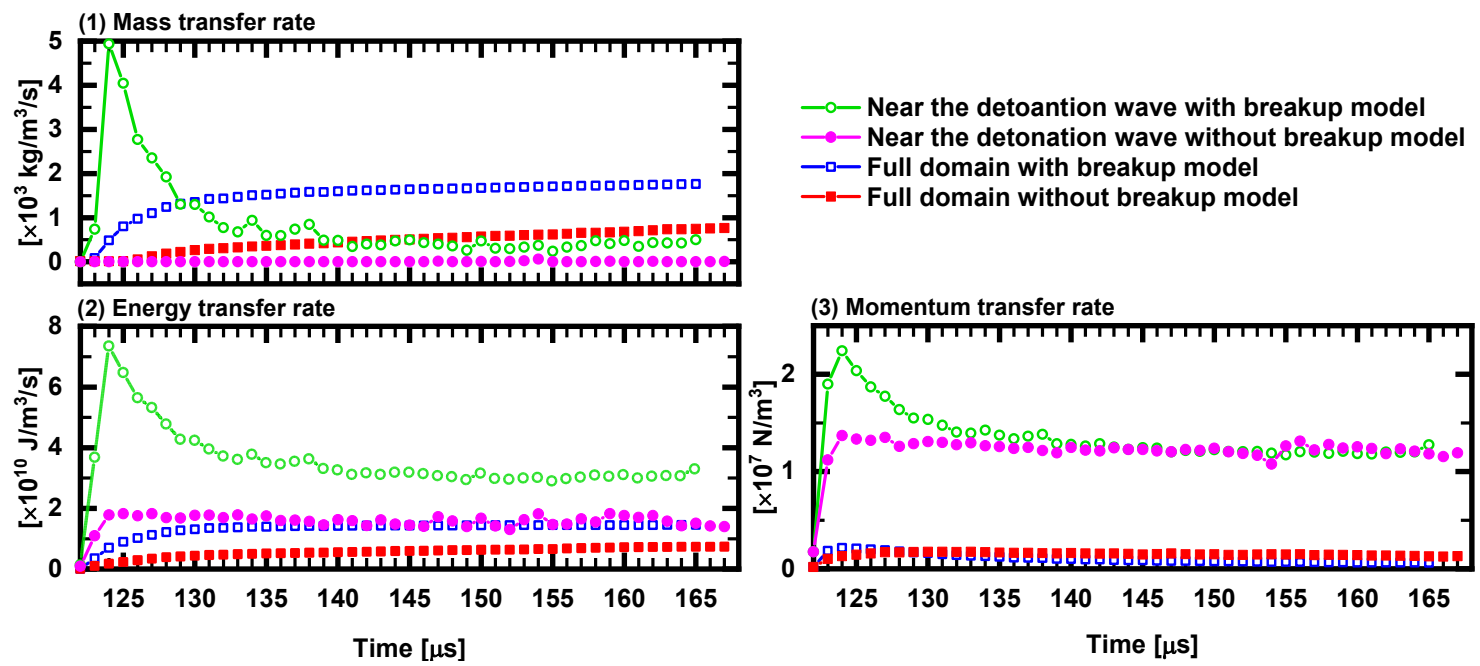

Figure 4. Time history of interphase exchange rates with or without breakup model: (1) mass, (2) energy and (3) momentum transfer rates. $d_{d}^{0}=10 \mu \mathrm{m}$ and $\mathrm{z}=0.31$

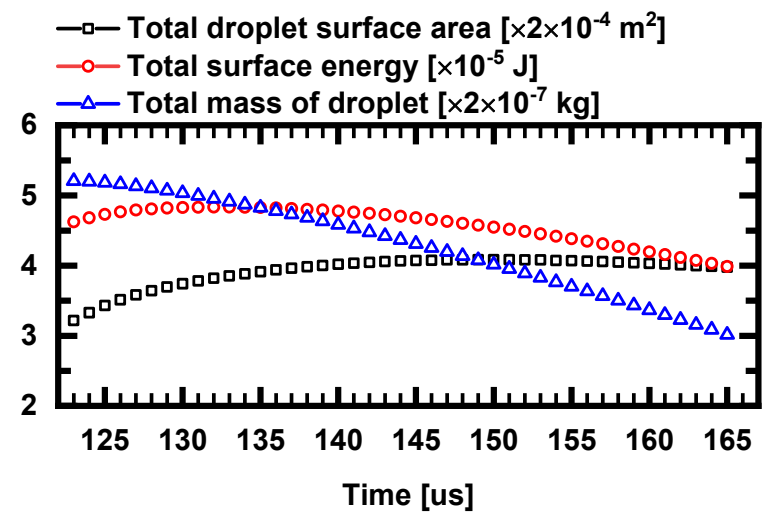

Figure 5. Evolution of total droplet surface and energy, as well as total mass of droplet.

The evolutions of volume-averaged interphase exchange rates in detonation propagation process with or without water droplet breakup are plotted in Figure 4. The results with breakup model deactivated are also added for comparisons. The time histories of total droplet surface area and energy [38], as well as total mass of water droplet, are also recorded in Figure 5. It is found that water droplet breakup increases the two interphase transfer rates: mass and energy exchange rates but have limited influence on momentum transfer rate. This is due to the breakup process promote transferring of mass and heat of water droplets. This conclusion is also demonstrated by the gradually increased total surface area in Figure 5, although the 
total mass and surface energy of water droplet is reduced. One can see from in Figure 4(1) that the mass transfer rate near the detonation wave peaks quickly after the detonation wave is transmitted into the two-phase section, and then reduces significantly but still larger than that without breakup model. This is because parent droplets disintegrate after entering the detonated area, and then more daughter droplets are evaporated. For the full domain cases, the evaporation rate increases gradually but is much smaller than that centring at the reaction front $5 \mathrm{~mm}$ before $129 \mu \mathrm{s}$. After the instant $130 \mu \mathrm{s}$, full domain with breakup model has the largest mass transfer rate. Thus, it can be concluded that mass transfer near the detonation wave covers small portion of mass exchanging in full domain. However, the energy transfer rate near the detonation wave is much larger than that the full domain. This is corresponding to the mainly detonative combustion heat release behind the detonation wave in Figure 4(c). Momentum transfer rate is less influenced by the droplet breakup for the full domain and near the detonation wave, and kinetic equilibrium is reached at the end, except the initial period of the case near the detonation wave, i.e., 122 - $130 \mu \mathrm{s}$. In addition, for the larger droplets, the momentum transfer rate is reduced compared with the smaller. This is because the larger droplets need longer droplet relaxation time. The droplet size effect on the interphase exchange rates will be studied in a future paper.
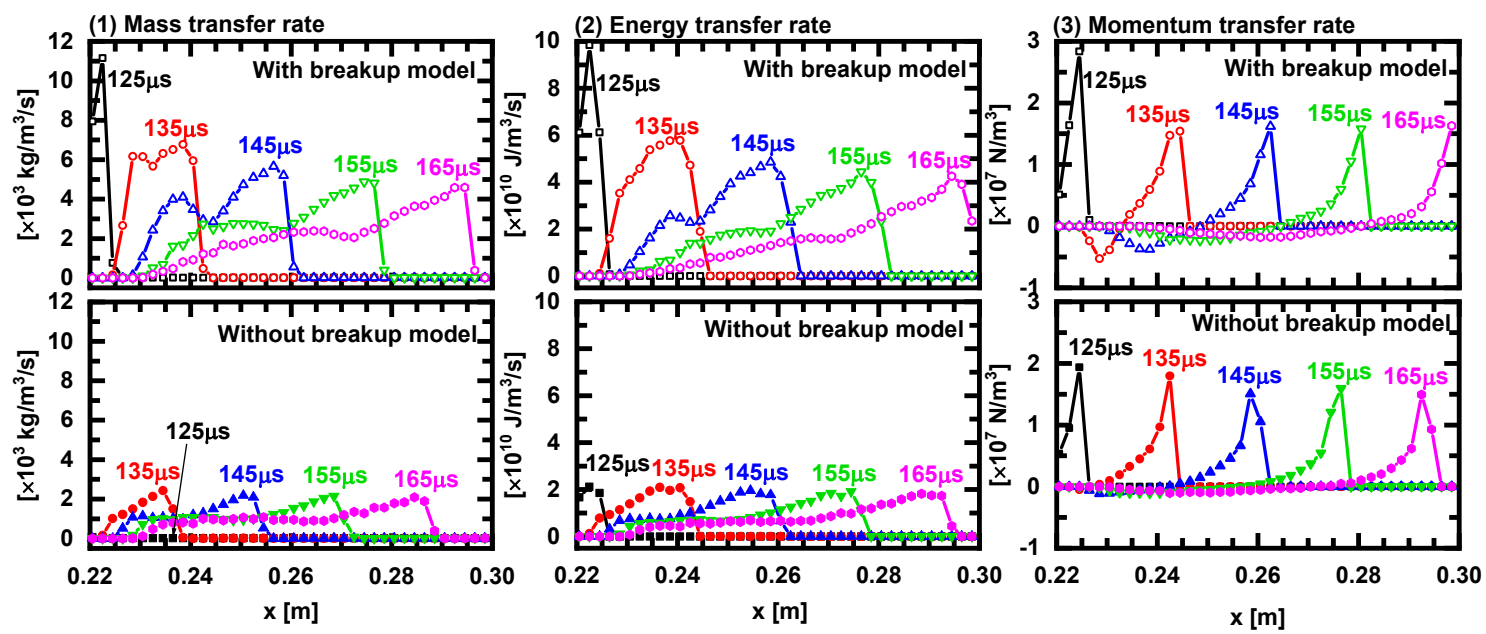

Figure 6. Profiles of averaged transfer rates of (a) mass, (b) energy and (c) momentum with or without breakup model at selected five instants.

To explore the droplet breakup effects, the temporal evolutions of width-averaged interphase exchange rates at selected five instants are presented in Figure 6. A positive mass (energy and momentum) transfer rate indicates that the transfer from liquid (gas) phase to gas (liquid) phase. In general, the water droplet disintegration promotes the average exchange rates of mass and heat, whilst it has less influence on momentum transfer rate. Note that peak values of exchange rates of mass and energy under breakup process is higher than that without droplet disintegration. The maximum transfer rates of mass and energy with breakup model decrease gradually, whilst the two exchange rates without droplet disintegration level off except droplet evaporation at the initial instant. It is also noticed that the negative momentum exchange rate appears in the downstream at 135 and 145 instants. This is implying that the momentum is transferred from the water droplets to the gas phase.

\section{Conclusions}


Effect of water droplet breakup on two-dimensional hydrogen/air detonations is computationally studied with a hybrid Eulerian-Lagrangian method considering two-way gasliquid coupling. General features of gas phase and liquid droplets are well captured. It is shown that, for the studied case, the water droplet breakup makes propagation of detonation wave more stable, compared with the case without breakup, and this is evidenced by the smaller and more regular cell size. The influence of water droplet disintegration on the hydrogen detonation is performed with the evolutions of the spatial distribution of interphase transfer rates (mass, energy, and momentum). The droplet breakup would have stronger influence on mass and energy transfer than the momentum transfer. It is also found that high interphase transfer rates of disintegration case are observed at the onset of detonation propagation, and they gradually decrease.

\section{Acknowledgments}

This work used the computational resources of ASPIRE 1 Cluster in The National Supercomputing Centre, Singapore (https://www.nscc.sg). Yong Xu is supported by the NUS Research Scholarship.

\section{References}

[1] Oran, E. S., Chamberlain, G., and Pekalski, A., 2020, Prog. Energy Combust. Sci., 77(77).

[2] Atkinson, G., Cowpe, E., Halliday, J., and Painter, D., 2017, J. Loss Prev. Process Ind., 48, pp. 367-375.

[3] Catlin, C., 2002, J. Hazard. Mater., 94(2), pp. 103-132.

[4] Thomas, G. O., 2000, Process Saf. Environ. Prot., 78(5), pp. 339-354.

[5] Grant, G., Brenton, J., and Drysdale, D., 2000, Prog. Energy Combust. Sci., 26(2), pp. 79-130.

[6] Zheng, R., Bray, K., and Rogg, B., 1997, Combust. Sci. Technol., 126, pp. 389-401.

[7] Song, Y., and Zhang, Q., 2019, J. Hazard. Mater., 363(June 2018), pp. 16-25.

[8] Boeck, L. R., Kink, A., Oezdin, D., Hasslberger, J., and Sattelmayer, T., 2015, Int. J. Hydrogen Energy, 40(21), pp. 6995-7004.

[9] Jourdan, G., Biamino, L., Mariani, C., Blanchot, C., Daniel, E., Massoni, J., Houas, L., Tosello, R., and Praguine, D., 2010, Shock Waves, 20(4), pp. 285-296.

[10] Chauvin, A., Jourdan, G., Daniel, E., Houas, L., and Tosello, R., 2011, Phys. Fluids, 23(11), pp. 1-13.

[11] Adiga, K. C., Willauer, H. D., Ananth, R., and Williams, F. W., 2009, Fire Saf. J., 44(3), pp. 363-369.

[12] Ananth, R., Willauer, H. D., Farley, J. P., and Williams, F. W., 2012, Fire Technol., 48(3), pp. 641-675.

[13] Schwer, D. A., and Kailasanath, K., 2007, Proc. Combust. Inst., 31 II, pp. 2361-2369.

[14] Thomas, G. O., Edwards, M. J., and Edwards, D. H., 1990, Combust. Sci. Technol., 71(4-6), pp. 233-245.

[15] Niedzielska, U., Kapusta, L. J., Savard, B., and Teodorczyk, A., 2017, J. Loss Prev. Process Ind., 50, pp. 229-236.

[16] Jarsalé, G., Virot, F., and Chinnayya, A., 2016, Shock Waves, 26(5), pp. 561-572.

[17] Watanabe, H., Matsuo, A., Matsuoka, K., Kawasaki, A., and Kasahara, J., 2019, Proc. Combust. Inst., 37(3), pp. 3617-3626.

[18] Watanabe, H., Matsuo, A., Chinnayya, A., Matsuoka, K., Kawasaki, A., and Kasahara, J., 2020, J. Fluid Mech., 887(887).

[19] Watanabe, H., Matsuo, A., Chinnayya, A., Matsuoka, K., Kawasaki, A., and Kasahara, J., 2020, Proc. Combust. Inst., 000, pp. 1-8.

[20] Macpherson, G. B., Nordin, N., and Weller, H. G., 2009, Commun. Numer. Methods Eng., 25(3), pp. 263-273.

[21] Crowe, C. T., Schwarzkopf, J. D., Sommerfeld, M., and Tsuji, Y., 1998, CRC Press, 
New York, U.S.

[22] Bradley RS, NABT-E, F., and GM, D. in, 1959, .

[23] Sazhin, S. S., 2006, Prog. Energy Combust. Sci., 32(2), pp. 162-214.

[24] Liu, A. B., Mather, D., and Reitz, R. D., 1993, SAE Tech. Pap., 1.

[25] Ranz, W. E., and Marshall, W. R., 1952, Chem. Eng. Prog., 48(3), pp. 141-146.

[26] Pilch, M., and Erdman, C. A., 1987, Int. J. Multiph. Flow, 13(6), pp. 741-757.

[27] Huang, Z., Zhao, M., Xu, Y., Li, G., and Zhang, H., 2021, Fuel, 286(P2), p. 119402.

[28] Zhao, M., Li, J. M., Teo, C. J., Khoo, B. C., and Zhang, H., 2020, Flow, Turbul. Combust., 104(1), pp. 261-290.

[29] Zhao, M., and Zhang, H., 2020, Fuel, 275(March), p. 117986.

[30] Zhao, M., and Zhang, H., 2021, Accept. by 38th Int. Symp. Combust. under Rev. by Proc. Combust. Inst.

[31] Zhao, M., and Zhang, H., 2020, Fuel, 280(June), p. 118534.

[32] Meng, Q., Zhao, M., Zheng, H., and Zhang, H., 2020, Fuel, 119808.

[33] Ó Conaire, M., Curran, H. J., Simmie, J. M., Pitz, W. J., and Westbrook, C. K., 2004, Int. J. Chem. Kinet., 36(11), pp. 603-622.

[34] Huang, Z., Zhao, M., Xu, Y., Li, G., and Zhang, H., 2020, Fuel, Under revi.

[35] Mahmoudi, Y., and Mazaheri, K., 2015, Acta Astronaut., 115, pp. 40-51.

[36] Mazaheri, K., Mahmoudi, Y., and Radulescu, M. I., 2012, Combust. Flame, 159(6), pp. 2138-2154.

[37] Yungster, S., and Radhakrishnan, K., 2004, Combust. Theory Model., 8(4), pp. 745770.

[38] Lefebvre, A. H., and McDonell, V. G., 2017, . 